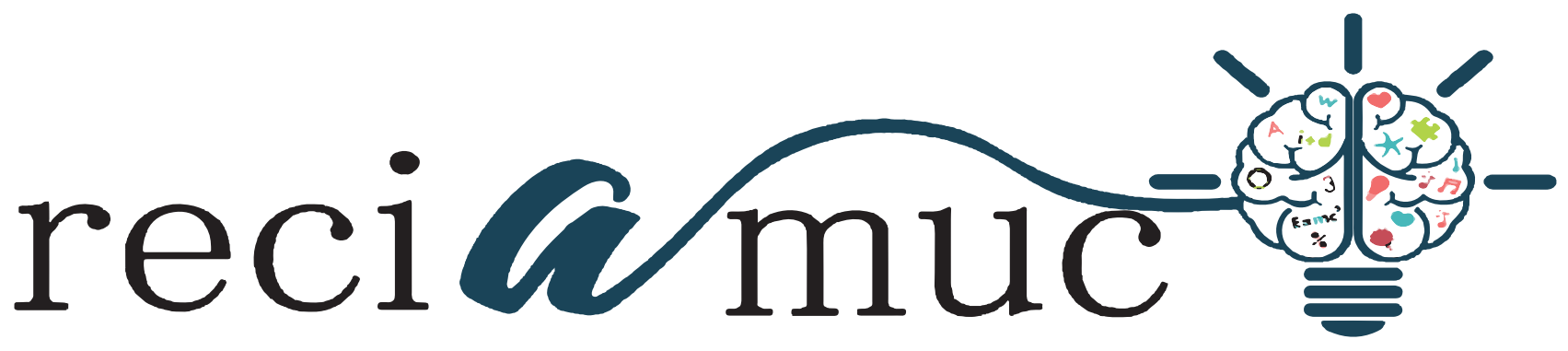

DOI: 10.26820/reciamuc/5.(3).agosto.2021.96-103

URL: https://reciamuc.com/index.php/RECIAMUC/article/view/696

EDITORIAL: Saberes del Conocimiento

REVISTA: RECIAMUC

ISSN: 2588-0748

TIPO DE INVESTIGACIÓN: Artículo de Revisión

CóDIGO UNESCO: 32 Ciencias Médicas

PAGINAS: 96-103

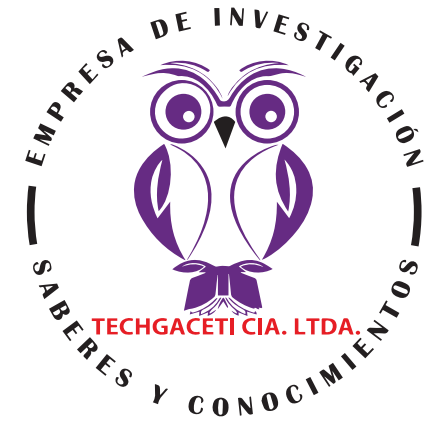

\title{
Dilema quirúrgico: perdida de habilidades quirúrgicas abiertas a cirugía mínimamente invasiva
}

Surgical dilemma: loss of surgical skills open to minimally

Complicações associadas à colecistectomia laparoscópiça de emergência

Karla Katherine Pérez Mendoza'; Sergio Geovanny Alvarado Muñoz; Juan Carlos González Jara ${ }^{3}$; Katerine Lilibeth Moreira Duran ${ }^{4}$

\section{RECIBIDO: 10/06/2021 ACEPTADO: 12/07/2021 PUBLICADO: 31/08/2021}

1. Licenciada en Enfermería de la Universidad Estatal de Milagro; Investigador Independiente; Milagro, Ecuador; karla.perez.mendoza.26@gmail.com; iD https://orcid.org/0000-0002-4115-0297

2. Médico de la Universidad de Guayaquil; Investigador Independiente; Guayaquil, Ecuador; geovanny18sergio@hotmail.com; iD https://orcid.org/0000-0002-8490-8096

3. Médico de la Universidad de Guayaquil; Investigador Independiente; Guayaquil, Ecuador; juancgj89@hotmail. com; iD https://orcid.org/0000-0003-0345-1459

4. Médico de la Universidad de Guayaquil; Investigador Independiente; Guayaquil, Ecuador; kathymodu22@ hotmail.com; (iD https://orcid.org/0000-0001-8526-1952

CORRESPONDENCIA

Karla Katherine Pérez Mendoza

karla.perez.mendoza.26@gmail.com

Guayaquil, Ecuador

C RECIAMUC; Editorial Saberes del Conocimiento, 2021 


\section{RESUMEN}

En los últimos 100 años la cirugía ha tenido un avance extraordinario, es cada vez más científica y su campo de investigación abre nuevos senderos. Continuamente se buscan nuevos procedimientos y nuevos abordajes desde cirugía mínimamente invasiva hasta abordajes virtuales a través de cirugía robótica. La cirugía es el término utilizado tradicionalmente para describir los procedimientos (llamados procedimientos quirúrgicos) que implican realizar incisiones o suturar tejidos para tratar enfermedades, lesiones o deformidades. La elección del tipo de cirugía a aplicar es fundamental para la adecuada exposición del campo quirúrgico que permita asegurar el control total del procedimiento realizado logrando el éxito de la cirugía y un posoperatorio favorable. Entre los principales tipos de cirugía se encuentran, la cirugía abierta, la cual tiene una serie de limitaciones y desventajas, entre las que resaltan que necesita incisiones muy amplias, existe mayor riesgo de infección, mayor dolor postoperatorio, mayor tiempo de reposo, recuperación más lenta y alta más tardía. Por su parte la cirugía mínimamente invasiva se realiza a través de incisiones muy pequeñas o sin necesidad de incisión, es una técnica quirúrgica que ha desplazado poco a poco a la cirugía abierta. El enfoque general del presente trabajo es dar a conocer el porqué del dilema quirúrgico por la pérdida de habilidades quirúrgicas abiertas a cirugía mínimamente invasiva, sus definiciones, tipos, ventajas, desventaja y como se proyecta este tipo de cirugía en el futuro. Los resultados se obtuvieron mediante el desarrollo de una investigación de tipo bibliográfica, que permite concluir que la perdida de habilidades quirúrgicas abiertas reside en las ventajas que la cirugía mínimamente invasiva tiene sobre la cirugía convencional, entre las que destacan, menor sangrado operatorio, menor dolor posquirúrgico y pronto restablecimiento de la vía oral, menor tiempo de hospitalización posquirúrgica, mejor efecto cosmético y calidad de vida, pronta reincorporación a la vida cotidiana, uso temprano de terapia adyuvante.

Palabras clave: Cirugía, dilema quirúrgica, procedimiento quirúrgico, cirugía abierta, cirugía mínimamente invasiva

\section{ABSTRACT}

In the last 100 years, surgery has had an extraordinary advance, it is increasingly scientific and its field of research opens new paths. New procedures and new approaches are continually being sought from minimally invasive surgery to virtual approaches through robotic surgery. Surgery is the term traditionally used to describe procedures (called surgical procedures) that involve making incisions or suturing tissue to treat disease, injury, or deformities. The choice of the type of surgery to be applied is essential for the adequate exposure of the surgical field to ensure total control of the procedure performed, achieving the success of the surgery and a favorable postoperative period. Among the main types of surgery are open surgery, which has a series of limitations and disadvantages, among which stand out that it needs very wide incisions, there is a greater risk of infection, greater postoperative pain, longer rest time, more recovery slower and higher later. For its part, minimally invasive surgery is performed through very small incisions or without the need for an incision, it is a surgical technique that has gradually displaced open surgery. The general approach of this work is to present the reason for the surgical dilemma due to the loss of surgical skills open to minimally invasive surgery, its definitions, types, advantages, disadvantages and how this type of surgery is projected in the future. The results were obtained through the development of a bibliographic type research, which allows to conclude that the loss of open surgical skills lies in the advantages that minimally invasive surgery has over conventional surgery, among which are less operative bleeding, less pain postoperative and early reestablishment of the oral route, shorter postoperative hospitalization time, better cosmetic effect and quality of life, prompt return to daily life, early use of adjuvant therapy.

Keywords: Surgery, surgical dilemma, surgical procedure, open surgery, minimally invasive surgery.

\section{RESUMO}

Nos últimos 100 anos, a cirurgia teve um avanço extraordinário, está cada vez mais científica e seu campo de pesquisa abre novos caminhos. Novos procedimentos e novas abordagens são continuamente buscados, desde a cirurgia minimamente invasiva até abordagens virtuais por meio da cirurgia robótica. Cirurgia é o termo tradicionalmente usado para descrever procedimentos (chamados procedimentos cirúrgicos) que envolvem fazer incisões ou suturar tecido para tratar doenças, lesões ou deformidades. A escolha do tipo de cirurgia a ser aplicada é essencial para a adequada exposição do campo cirúrgico para garantir total controle do procedimento realizado, obtendo-se o sucesso da cirurgia e um pós-operatório favorável. Dentre os principais tipos de cirurgia estão a cirurgia aberta, que apresenta uma série de limitações e desvantagens, entre as quais se destacam que necessita de incisões muito largas, há maior risco de infecção, maior dor pós-operatória, maior tempo de descanso, maior recuperação mais lenta e mais tarde. Por sua vez, a cirurgia minimamente invasiva é realizada através de incisões muito pequenas ou sem a necessidade de uma incisão, é uma técnica cirúrgica que tem gradualmente deslocado a cirurgia aberta. A abordagem geral deste trabalho é apresentar a razão do dilema cirúrgico pela perda de habilidades cirúrgicas abertas à cirurgia minimamente invasiva, suas definições, tipos, vantagens, desvantagens e como esse tipo de cirurgia se projeta no futuro. Os resultados foram obtidos por meio do desenvolvimento de uma pesquisa do tipo bibliográfica, que permite concluir que a perda das habilidades cirúrgicas abertas reside nas vantagens que a cirurgia minimamente invasiva tem sobre a cirurgia convencional, entre as quais estão menos sangramento operatório, menos dor pós-operatória e restabelecimento precoce. da via oral, menor tempo de internação pós-operatória, melhor efeito cosmético e qualidade de vida, pronto retorno à vida diária, uso precoce de terapia adjuvante.

Palavras-chave: Cirurgia, dilema cirúrgico, procedimento cirúrgico, cirurgia aberta, cirurgia minimamente invasiva. 


\section{Introducción}

La Cirugía o procedimiento quirúrgico, "es la rama de la medicina que previene, cura o rehabilita enfermedades en pacientes cortando, separando, reparando o sustituyendo tejidos u órganos mediante instrumentos, generalmente bajo anestesia. Es una disciplina eminentemente práctica, enfocada en la acción." (Porras, 2016).

"En los últimos 100 años la cirugía ha tenido un avance extraordinario, es cada vez más científica y su campo de investigación abre nuevos senderos. Continuamente se buscan nuevos procedimientos y nuevos abordajes desde cirugía mínimamente invasiva hasta abordajes virtuales a través de cirugía robótica." Baridó et al. (2012).

Entre los tipos de cirugía usados con más frecuencia están la cirugía abierta o convencional y la cirugía mínimamente invasiva, definidas por Roldán (2020):

- La cirugía abierta: tiene una serie de limitaciones y desventajas: necesita incisiones muy amplias, habitualmente $>5 \mathrm{~cm}$ y depende del órgano a tratar; mientras más amplia la incisión mejor visibilidad. Existe mayor riesgo de infección, mayor dolor postoperatorio, mayor tiempo de reposo, recuperación más lenta y alta más tardía.

- La cirugía mínimamente invasiva: se realiza a través de incisiones muy pequeñas o sin necesidad de incisión, es una técnica quirúrgica que ha desplazado poco a poco a la cirugía abierta.

El dilema quirúrgico por la pérdida de habilidades quirúrgicas abiertas debido a la cirugía mínimamente invasiva reside en las ventajas que esta última tiene sobre la cirugía convencional, entre las que destacan las indicadas por Chumpitaz (2011):

La cirugía mínimamente invasiva (laparoscopia) es una técnica quirúrgica de acceso mínimo, con excelentes resultados, disminuyendo las complicaciones de la cirugía tradicional, siendo los más evidente, la infección de la herida operatoria así como el dolor postoperatorio. Es un excelente método inicial de diagnóstico y se prefiere como tal, y después del diagnóstico, ofrece la posibilidad de resolución por esta vía.

El objetivo de este trabajo investigativo, se centra en abordar el dilema quirúrgico de la perdida de habilidades quirúrgicas abiertas a cirugía mínimamente, todo ello con la finalidad de proporcionar un material actualizado que defina dichos aspectos y que sea de utilidad no solo para el personal de salud, sino también para el público en general, por lo que para ello se estructura de la siguiente manera: definiciones, tipos de cirugías, principales ventajas y desventajas y futuro de las cirugías.

\section{Materiales y métodos}

Para desarrollar la presente investigación se buscó material bibliográfico digitalizado, el cual representó los cimientos del análisis y compendio del presente tema. Por lo tanto, la presente investigación es de tipo documental o bibliográfica.

La búsqueda se realizó a través de varias bases de datos y de páginas web implicadas con el área de la salud, con amplio reconocimiento científico a nivel mundial. Entre las bases de datos que se utilizaron se encuentran: SciELO, Redalyc, Medigraphic, entre otros. Asimismo, fueron usados los portales de la Clínica Mayo, MedlinePlus, entre otras.

El tipo de material seleccionado consistió en informes, tesis de grado, protocolos y otras clases de contenidos. Fueron excluidos aquellos contenidos repetidos, las editoriales o cartas editoriales, anotaciones académicas y todo documento carente de sustento científico o bajo nivel de evidencia.

El tiempo de búsqueda se llevó a cabo durante mediados del mes agosto del presente año. En cuanto a los descriptores, fueron usaron los siguientes: "cirugía abierta", "ci- 
rugía mínimamente invasiva"; "dilema quirúrgico: perdida de habilidades quirúrgicas abiertas". Los resultados hallados fueron filtrados bajo criterios como idioma español. Asimismo, se seleccionaron trabajos con mayor relevancia y con correlación temática. Los trabajos seleccionados tuvieron una fecha de publicación de 2010 al 2021.

Por último, el equipo investigador destaca que no hubo conflicto de intereses.

\section{Resultados}

\section{Cirugía o Procedimiento Quirúrgico}

El comienzo de la cirugía se remonta a los anales de la historia del propio ser humano, sin embargo, debemos diferenciar el punto de inflexión que marca un antes y un después en la práctica quirúrgica, y sobre el cual Alcalá et al. (2012) indican que:

Este punto de inflexión se produjo en el siglo XIX, donde el descubrimiento de la anestesia en primer término, y de la antisepsia posteriormente constituyeron dos verdaderos hitos que habrían de catapultar a la cirugía para alcanzar los niveles de especialización de los que hoy gozamos tan solo 150 años después.

La cirugía "es el término utilizado tradicionalmente para describir los procedimientos (llamados procedimientos quirúrgicos) que implican realizar incisiones o suturar tejidos para tratar enfermedades, lesiones o deformidades." (Mohabir \& Coombs, 2020)

\section{Tipos de Cirugía}

La elección del tipo de cirugía a aplicar "es fundamental para la adecuada exposición del campo quirúrgico que permita asegurar el control total del procedimiento realizado logrando el éxito de la cirugía y un posoperatorio favorable." Ramírez et al. (2016)

Una cirugía no siempre implica cortes (incisiones) grandes y largos períodos de recuperación, como en el pasado. Dependiendo del tipo de cirugía, pueden usarse diversos métodos, entre los que destacan los referi- dos por De Muro et al. (2020):

- Cirugía abierta: significa cortar la piel y los tejidos para que el cirujano pueda ver completamente las estructuras y los órganos involucrados.

- Cirugía mínimamente invasiva: En esta cirugía, no es necesario hacer una incisión grande. Este método ofrece una recuperación más rápida y con menos dolor que la cirugía abierta. No todas las afecciones son adecuadas para este método. Puede usarse no solo para evaluar enfermedades y lesiones, sino también para tomar muestras de tejido y hacer reparaciones. Este tipo de cirugía incluye las siguientes técnicas:

- Laparoscopía. Se usa un tubo con una luz y una cámara en el extremo (laparoscopio) para revisar los órganos del abdomen. Suele usarse para mirar dentro del abdomen sin la necesidad de hacer incisiones grandes. También se pueden tomar muestras de tejido para analizarlas.

- Endoscopia. En esta prueba se usa un tubo pequeño y flexible con una luz y una cámara en el extremo (endoscopio) para revisar el interior de los órganos huecos del tubo digestivo. También se pueden tomar muestras de tejido para analizarlas. La endoscopia puede usarse en el estómago (gastroscopia), el esófago (esofagoscopia) y el colon (sigmoidoscopia o colonoscopia).

- Artroscopia. Se usa para mirar el interior de una articulación con un tubo llamado artroscopio. Este método suele usarse para revisar y quizás reparar el interior de una rodilla, hombro o articulación de la cadera.

- Broncoscopia. Se usa para examinar las principales vías respiratorias de los pulmones (bronquios) con un tubo rígido o flexible (broncoscopio). 
La broncoscopia ayuda a evaluar y diagnosticar problemas en los pulmones. También ayuda a evaluar obstrucciones, tomar muestras de tejido o líquido, o extirpar un cuerpo extraño.

- Toracoscopia. Esta es una cirugía mínimamente invasiva en la cavidad del pecho.

- Cistoscopia. Mediante este método, se coloca un tubo de visualización para mirar la uretra y la cavidad de la vejiga.

- Histeroscopia. Se usa para revisar el conducto endocervical y la cavidad uterina con un endoscopio.

- Laringoscopia. Se usa para revisar la laringe con un espejo o tubo de visualización.

La cirugía abierta "es la cirugía convencional, se basa en exponer para poder ver el problema del paciente, se realizan los gestos quirúrgicos necesarios, se corrige el problema y para finalizar, en la cirugía abierta simplemente se dan unos puntos de sutura para volver a cerrar las capas." (Instituto de Biomecánica \& Cirugía, 2020)

Las innovaciones continuas en la cirugía mínimamente invasiva proporcionan beneficios para los pacientes que sufren una gran variedad de enfermedades. Al respecto la Clínica Mayo (2021) clasifica este tipo de cirugía de la siguiente manera:

- Laparoscopia: una cirugía que se realiza a través de una o más incisiones pequeñas con tubos pequeños, y cámaras e instrumentos quirúrgicos diminutos, fue uno de los primeros tipos de cirugía mínimamente invasiva.

- Cirugía robótica. Proporciona una visión 3D amplificada del área de la cirugía y ayuda al cirujano a operar con precisión, flexibilidad y control.

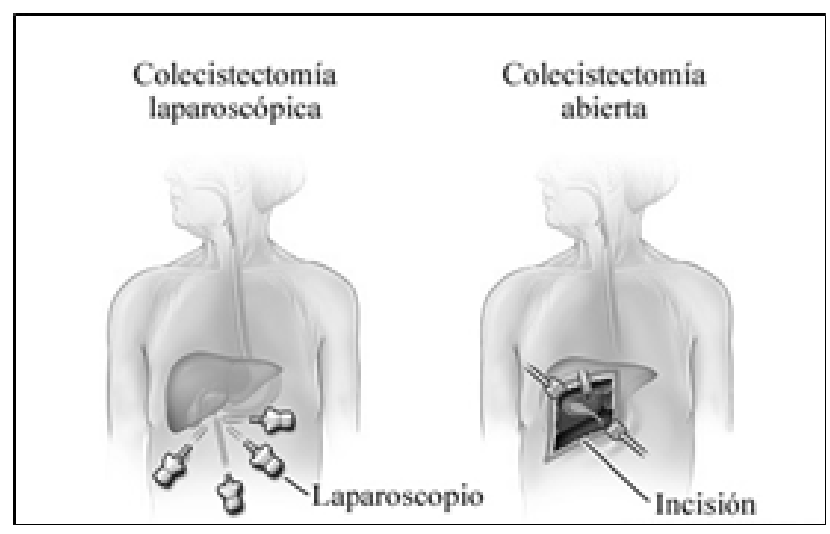

Figura 1. Tipos de cirugía. Recuperado de:

"Tipos de cirugía para extirpar la vesícula

$$
\text { biliar". }
$$

Fuente: Healthwise. (2021). URL: https:// www.cigna.com/es-us/individuals-families/ health-wellness/hw/tipos-de-ciruga-para-extirpar-la-vescula-biliar-zm2671

\section{Ventajas de la cirugía mínimamente inva- siva sobre la cirugía abierta}

En las últimas décadas, la cirugía mínimamente invasiva ha evolucionado. Mientras que en sus comienzos era considerada solo una herramienta de diagnóstico, actualmente se emplea como método quirúrgico. Entre los beneficios que este tipo de cirugía ofrece respecto a la cirugía convencional Valdivia et al. (2013) destacan:

- Menor sangrado operatorio.

- Menor dolor posquirúrgico y pronto restablecimiento de la vía oral.

- Menor tiempo de hospitalización posquirúrgica.

- Mejor efecto cosmético y calidad de vida.

- Pronta reincorporación a la vida cotidiana.

- Uso temprano de terapia adyuvante.

Por su parte Layunta et al. (2018) indican que en los últimos años, la cirugía mínimamente invasiva ha conseguido reducir las 
complicaciones quirúrgicas propias de la cirugía abierta, destacando como principales ventajas las siguientes:

- Realización de incisiones cutáneas más pequeñas.

- Menor tasa de infección de la herida: debido a una menor exposición de los órganos internos al entorno externo.

- Menor hemorragia intraoperatoria: y, por tanto, un menor uso de transfusiones de sangre.

- Menor dolor postoperatorio en el primer día: esto conduce a una menor utilización de analgésicos postoperatorios.

- Reducción de la estancia hospitalaria.

- Menor costo económico: aunque el coste del proceso quirúrgico por vía laparoscópica es más elevado, este tipo de intervención a la larga supone menor gasto económico debido a la menor utilización de fármacos así como estancias hospitalarias más cortas.

\section{Futuro de la cirugía}

A medida que las tecnologías mejoran, las máquinas inteligentes y autónomas pueden obtener la capacidad de aumentar o superar a los seres humanos en tareas operativas y de toma de decisiones. Según (Díaz, 2021):

Las innovaciones en cirugía mínimamente invasiva y toma de decisiones quirúrgicas han mejorado las capacidades de los cirujanos para realizar operaciones y ejercer un juicio sólido. A medida que las tecnologías mejoran, estas innovaciones dependen menos de la aportación humana y más de las máquinas inteligentes y autónomas, es decir, de los sistemas informáticos que aprenden a realizar tareas humanas y funciones cognitivas con cierto grado de independencia.
De la mano de la tecnología, el mundo está cambiando y vemos cada día progresos que nos maravillan. Estos avances han impactado en los procedimientos quirúrgicos tradicionales, tal y como lo refiere Giménez (2017):

El futuro de la cirugía estará relacionado con la inteligencia artificial, la robótica, el "data-driven" y el avance de las imágenes en cirugía (calidad de imagen, fusión de imágenes y guías de localización). Estos avances requieren una reconversión del cirujano, que deberá adoptar además diversas habilidades no técnicas, con una profunda impronta en el trabajo en equipo y ejercitando nuevas maneras de liderazgo.

Lo antes expuesto, evidencia que "el advenimiento de la cirugía mínimamente invasiva representa un peldaño particularmente grande en una serie de avances médicos y tecnológicos como los nuevos aparatos de energía para disección y hemostasia, las técnicas de imagenología avanzada y la cibernética médica, que contribuyen con el bienestar de los pacientes." García et al. (2016)

\section{Conclusiones}

En las últimas décadas el avance tecnológico en cirugía y la implantación de la cirugía mínimamente invasiva no solo como método diagnostico sino como procedimiento quirúrgico ha generado grandes cambios y la necesidad de adaptarse a ellos, ya que ha modificado radicalmente la forma de dar atención a los enfermos. La cirugía de invasión mínima al igual que la cirugía llamada tradicional o abierta, pretende buscar un beneficio claro y definitivo para el paciente.

Debido a que la vía de acceso y las incisiones son menores de tamaño, éstas causarán, obviamente menor dolor, la cirugía mínimamente invasiva ha ido desplazando poco a poco y no solamente observamos estos beneficios, sino que también, si partimos del mismo concepto de causar menor daño posible en nuestro enfermo, al haber 
incisiones de menor tamaño, su recuperación será más rápida y por lo tanto podrá incorporarse a sus actividades familiares, sociales tanto podrá incorporarse a sus actividades familiares, sociales y laborales en menor tiempo que con los procedimientos quirúrgicos tradicionales, con los consiguientes beneficios personales y económicos que esto implica.

\section{Bibliografía}

Alcalá, J., Alcalá, A., Ahumada, J., \& Morilla, M. (2012). BREVE HISTORIA DE LA CIRUGÍA. HITOS EN EL DESARROLLO DE LA CIRUGÍA MODERNA. Revista Española de Podología, 23(5), 176-182. Recuperado el 14 de 08 de 2021, de https://www.elsevier.es/es-revista-revista-espanola-podologia-224-articulo-breve-historia-de-la-cirugia--X0210123812502807

Baridó, M., De La Torre, A., \& Macias, A. (01 de 09 de 2012). Evolución de la cirugía: La meta es lograr procedimientos seguros. Revista UNAM, 13(9). Recuperado el 13 de 08 de 2021, de http://www. revista.unam.mx/vol.13/num9/art92/

Chumpitaz, K. (2011). Universidad Ricardo Palma. Recuperado el 11 de 08 de 2021, de https://repositorio.urp.edu.pe/bitstream/handle/urp/237/ chumpitaz_k.pdf?sequence $=1$ \&isAllowed $=y$

Clinica Mayo. (29 de 07 de 2021). Clinica Mayo. Recuperado el 12 de 08 de 2021, de Clinica Mayo: https://www.mayoclinic.org/es-es/tests-procedures/ minimally-invasive-surgery/about/pac-20384771

De Muro, J., Fraser, M., \& Foley, M. (12 de 01 de 2020). UC San Diego Health. Recuperado el 14 de 08 de 2021, de UC San Diego Health: https://myhealth. ucsd.edu/Spanish/Relatedltems/85,P04501

Díaz, C. (07 de 05 de 2021). Gestión y Economía de la Salud por Carlos Diaz. Recuperado el 13 de 08 de 2021, de https://saludbydiaz.com/2021/05/07/ el-futuro-de-la-cirugia/

Garcia, A., Gutierrez, L., \& Cueto, J. (06 de 2016). Evolución histórica de la cirugía laparoscópica. Asociación Mexicana de Cirugía Endoscópica, A.C., 17(2), 93-106. Recuperado el 15 de 08 de 2021, de https://www.medigraphic.com/pdfs/endosco/ce-2016/ce162i.pdf

Giménez, M. (07 de 2017). La cirugía del futuro. Acta Gastroenterológica Latinoamericana, 47(2), 92-98. Recuperado el 15 de 08 de 2021, de https://www. redalyc.org/pdf/1993/199352110001.pdf
Healthwise. (10 de 02 de 2021). Cigna. Recuperado el 13 de 08 de 2021, de Cigna: https://www.cigna.com/es-us/individuals-families/health-wellness/ hw/tipos-de-ciruga-para-extirpar-la-vescula-biliar-zm2671

Instituto de Biomecánica \& Cirugía. (07 de 01 de 2020). Instituto de Biomecánica \& Cirugía. Recuperado el 15 de 08 de 2021, de Instituto de Biomecánica \& Cirugía: https://biomecanicavitruvio. com/2020/01/07/cirugia-abierta-vs-cirugia-mis/

Layunta, M., Luna, C., Remacha, A., \& Lopez, C. (12 de 05 de 2018). Ventajas de la cirugía laparoscópica sobre la cirugía abierta en la apendicitis aguda. Revista Electrónica Portales Médicos, 13(8), 538. Recuperado el 11 de 08 de 2021, de https://www. revista-portalesmedicos.com/revista-medica/ventajas-de-la-cirugia-laparoscopica-sobre-la-cirugia-abierta-en-la-apendicitis-aguda/

Mohabir, P., \& Coombs, A. (12 de 2020). Manual MSD. Recuperado el 12 de 08 de 2021, de Manual MSD: https://www.msdmanuals.com/es-ve/hogar/ temas-especiales/cirug\%C3\%ADa/cirug\%C3\%A$\mathrm{Da}$

Porras, J. (12 de 2016). Enseñanza y aprendizaje de la cirugía. Investigación en educación médica, 5(20). doi:https://doi.org/10.1016/j. riem.2016.05.008

Ramírez, J., Werner, K., Court, F., \& Sepúlveda, R. (2016). Universidad de Los Andes. Recuperado el 15 de 08 de 2021, de Universidad de Los Andes: https://www.uandes.cl/wp-content/uploads/2020/02/ Manual-de-Cirugia-UANDES.pdf

Roldan, F. (16 de 07 de 2020). Uros Associat. Recuperado el 14 de 08 de 2021, de https://urosassociats.com/ventajas-de-la-cirugia-minimamente-invasiva-sobre-la-cirugia-convencional/

Valdivia, H., Zevallos, A., Alvarez, M., Santos, C., \& Sanchez, M. (12 de 2013). La cirugía mínimamente invasiva evolución y su aplicación en ginecología oncológica. Revista Peruana de Ginecología y Obstetricia, 59(4). Recuperado el 11 de 08 de 2021 , de http://www.scielo.org.pe/scielo.php?script=sci_arttext\&pid=\$2304-51322013000400008 


\section{CitAR ESTE ARTICULO:}

Pérez Mendoza, K. K., Alvarado Muñoz, S. G., González Jara, J. C., \& Moreira Duran, K. L. (2021). Dilema quirúrgico: perdida de habilidades quirúrgicas abiertas a cirugía mínimamente invasiva. RECIAMUC, 5(3), 96-103. https:// doi.org/10.26820/reciamuc/5.(3).agosto.2021.96-103 MERCIAL-COMPARTIRIGUAL 4.0 . 